\title{
Adiabatic Density Perturbations and Matter Generation from the Minimal Supersymmetric Standard Model
}

\author{
Kari Enqvist, ${ }^{1,2}$ Shinta Kasuya, ${ }^{2}$ and Anupam Mazumdar ${ }^{3}$ \\ ${ }^{1}$ Department of Physical Sciences, P.O. Box 64, FIN-00014, University of Helsinki, Helsinki, Finland \\ ${ }^{2}$ Helsinki Institute of Physics, P.O. Box 64, FIN-00014, University of Helsinki, Helsinki, Finland \\ ${ }^{3}$ Physics Department, McGill University, 3600-University Road, Montreal, H3A 2T8, Canada
}

(Received 12 November 2002; published 6 March 2003)

\begin{abstract}
We propose that the inflaton is coupled to ordinary matter only gravitationally and that it decays into a completely hidden sector. In this scenario both baryonic and dark matter originate from the decay of a flat direction of the minimal supersymmetric standard model, which is shown to generate the desired adiabatic perturbation spectrum via the curvaton mechanism. The requirement that the energy density along the flat direction dominates over the inflaton decay products fixes the flat direction almost uniquely. The present residual energy density in the hidden sector is typically shown to be small.
\end{abstract}

PACS numbers: $98.80 . \mathrm{Cq}, 12.60 . \mathrm{Jv}$

Recent observations of the cosmic microwave background [1-3] appear to strongly support the inflationary paradigm. The Universe is flat and the perturbation spectrum is adiabatic, as suggested by the simplest inflaton models; in general, there could be a sizable isocurvature contribution, too (see, e.g., [4]). However, despite their many successes, these models leave open the origin of ordinary baryonic matter. Indeed, there are no concrete particle physics models that would specify how the inflaton couples to the standard model (SM) degrees of freedom. Since the inflaton should be a SM gauge singlet field, it can couple to quarks and leptons only indirectly via some other fields, or by some nonrenormalizable couplings. Such couplings are always rather ad hoc.

Therefore the most natural assumption would appear to be that the inflaton lies in a completely hidden sector and hence, except for gravity, does not couple to the SM degrees of freedom at all. Under such a circumstance, the inflaton decays entirely into lighter particles in the hidden sector, and the SM matter cannot originate from the inflaton energy density, as is usually assumed. In principle, there could be some other hidden degrees of freedom which might scatter to form SM particles. However, the scattering rate for particles coupled gravitationally to SM remains less than the Hubble rate until the temperature of the Universe drops to $T \lesssim\left(m_{\text {inf }} / M_{\mathrm{P}}\right)^{4} M_{\mathrm{P}} \sim$ $300 \mathrm{keV}$, where $m_{\mathrm{inf}}$ is the inflaton mass, and $M_{\mathrm{P}} \simeq$ $2.4 \times 10^{18} \mathrm{GeV}$ is the reduced Planck mass. Here we simply assumed that $m_{\mathrm{inf}}<10^{13} \mathrm{GeV}$, which holds, e.g., in chaotic inflation. This is a too low temperature for big bang nucleosynthesis (BBN) to be successful. At such low temperatures baryogenesis and the generation of cold dark matter (CDM) would also be a great problem.

In this Letter we present a simple mechanism to create the ordinary SM particles in the universe from a flat direction of minimal supersymmetric standard model (MSSM) along which, in the absence of supersymmetry breaking and nonrenormalizable operators, the scalar potential vanishes. The MSSM flat directions have all been classified in [5]. They are made of certain combinations of the squarks and sleptons, or the Higgs field (for a review of cosmological aspects of MSSM flat directions, see [6]). It is thus obvious that the decay of the field corresponding to the flat direction can give rise to the quarks and leptons, together with CDM in the form of lightest supersymmetric particles (LSPs). Moreover, as we will show, the same flat direction can provide the adiabatic fluctuations seeding the large scale structures in the universe.

Our starting point is thus inflation which takes place in a hidden sector. For a successful inflation, the energy density along the MSSM flat directions should be negligible during inflation. There will, however, be field fluctuations along those flat directions, powered by the nonzero energy density of the inflaton; these will be isocurvature in nature [7,8] (see also [9]). We may assume that within the single horizon volume which is inflated to become the observable universe, only one of the many flat directions is chosen. After the inflaton has decayed to light degrees of freedom (in the hidden sector), the flat direction energy density may nevertheless become dominant at a later time, and as a consequence the original isocurvature fluctuations turn adiabatic. Such a conversion of isocurvature fluctuations to adiabatic fluctuations has been dubbed as the curvaton scenario in [10], and has been previously considered in the context of prebig bang model [11] (for recent discussions on curvaton, see [12]).

The crucial requirement for an MSSM flat direction to act as a curvaton is that it does not receive a Hubbleinduced mass term during inflation. This is known to hold true at least in D term inflation [13] and generically in theories with "Heisenberg symmetry" [14]. In the latter case a one-loop contribution eventually gives a Hubbleinduced mass correction to the flat directions (other than stops) of order $10^{-1} H$ [14], where $H$ is the Hubble rate during inflation. There might be other possibilities, too. 
Let us here just assume that no mass term is induced by the hidden sector inflaton $I$ and that the energy scale of the inflaton is $V_{I}=\xi^{4}$. After inflation the hidden sector inflaton starts to oscillate, eventually decaying into light hidden degrees of freedom which are coupled to MSSM only gravitationally. As a consequence, there will be no thermalization between the light hidden degrees of freedom and the MSSM degrees of freedom, although in general there will be a nonzero temperature in the hidden sector.

The MSSM flat direction is described by a scalar field whose potential vanishes along that direction. However, the flatness will be lifted by supersymmetry breaking. It will also be lifted by nonrenormalizable terms of the form $W=\lambda \Phi^{n} / n M^{n-3}$ [5,15], where $M$ is a cutoff scale and $n \geq 4$ is the dimensionality of the nonrenormalizable operator; for each flat direction, there exists a set of allowed nonrenormalizable operators. In general, the flat direction potential can be written as [16]

$$
V(\phi)=\frac{1}{2} m_{\phi}^{2} \phi^{2}+\frac{\lambda^{2} \phi^{2(n-1)}}{2^{n-1} M^{2(n-3)}},
$$

where $\Phi=\phi e^{i \theta} / \sqrt{2}$ and the first term comes from supersymmetry breaking so that $m_{\phi} \sim \mathrm{TeV}$.

After inflation, the amplitude of the flat direction is very large, and $\phi$ will start to oscillate, eventually decaying. If the effective mass of the decay product is heavier than the mass of the flat direction, $g \phi>m_{\phi}$, where $g$ is some gauge or Yukawa coupling, it can decay into light particles only through loop diagrams involving heavy particles. On the other hand, the flat direction will decay at tree level if $f \phi<m_{\phi}$, where $f$ is some gauge or Yukawa coupling. In this case, the decay rate is given by

$$
\Gamma_{\phi} \sim \frac{f^{2} m_{\phi}}{8 \pi} .
$$

Since the flat direction is assumed to dominate the energy density of the universe when it decays so that $H \sim$ $m_{\phi} \phi / M_{\mathrm{P}}$, the amplitude of the flat direction at that time is given by

$$
\phi_{d} \sim f^{2} m_{\phi} .
$$

The Hubble parameter at the time of decay reads as

$$
H_{d} \sim f^{2} m_{\phi} \lesssim 10^{-10} m_{\phi},
$$

since $f<\left(m_{\phi} / M_{\mathrm{P}}\right)^{1 / 3} \sim 10^{-5}$ because $f \phi_{d}<m_{\phi}$. This roughly yields a temperature of order $10^{5} \mathrm{GeV}$, hence giving rise to a hot big bang universe in the SM sector. This observable sector reheat temperature is high enough for baryogenesis to take place at the electroweak phase transition [17] as well as for the LSPs to have the right (thermal) abundance required for CDM [18].

Let us now follow the dynamics of the flat direction during and after inflation until its decay. During inflation, the energy is dominated by the inflaton, and the flat direction quickly gets into the slow roll regime. The amplitude during inflation is given by

$$
\phi=\phi_{\mathrm{sr}} \sim\left(\frac{\xi^{2} M^{n-3}}{\lambda M_{\mathrm{P}}}\right)^{1 /(n-2)} .
$$

The amplitude of the (isocurvature) fluctuation of the flat direction, which will be converted to the adiabatic fluctuation at a later time, is expected to be $P_{\zeta}^{1 / 2} \sim$ $H_{\text {inf }} / \phi_{\text {sr }} \sim 10^{-5}$, so that the scale of inflation in the hidden sector should be

$$
\xi^{4} \sim\left(P_{\zeta}^{n-2} \lambda^{-2}\right)^{1 /(n-3)}\left(\frac{M}{M_{\mathrm{P}}}\right)^{2} M_{\mathrm{P}}^{4}
$$

Since the adiabatic fluctuation from the inflaton field is assumed to be a subdominant component in our scenario, the energy scale of inflation is observationally restricted by $\xi \ll 3 \times 10^{-2} \epsilon^{1 / 4} M_{\mathrm{P}}$ [10], where $\epsilon=$ $\left[V_{I}^{\prime}(I) / V_{I}\right]^{2} M_{\mathrm{P}}^{2} / 2$ is the slow roll parameter [4], and the prime denotes a derivative with respect to the inflaton field $I$.

During inflation the inflaton energy dominates the universe so that the ratio

$$
\left.\frac{V_{\phi}}{V_{I}}\right|_{\phi_{\mathrm{sr}}} \sim\left[\lambda^{-1} P_{\zeta}^{1 / 2}\left(\frac{M}{M_{\mathrm{P}}}\right)^{n-3}\right]^{2 /(n-3)}
$$

will be much less than unity.

The evolution of the energy densities of both the inflaton and the flat direction is shown schematically in Fig. 1. After inflation, the inflaton field oscillates around the minimum of its potential and its energy density decreases as $a(t)^{-3}$, where $a(t)$ is the scale factor of the

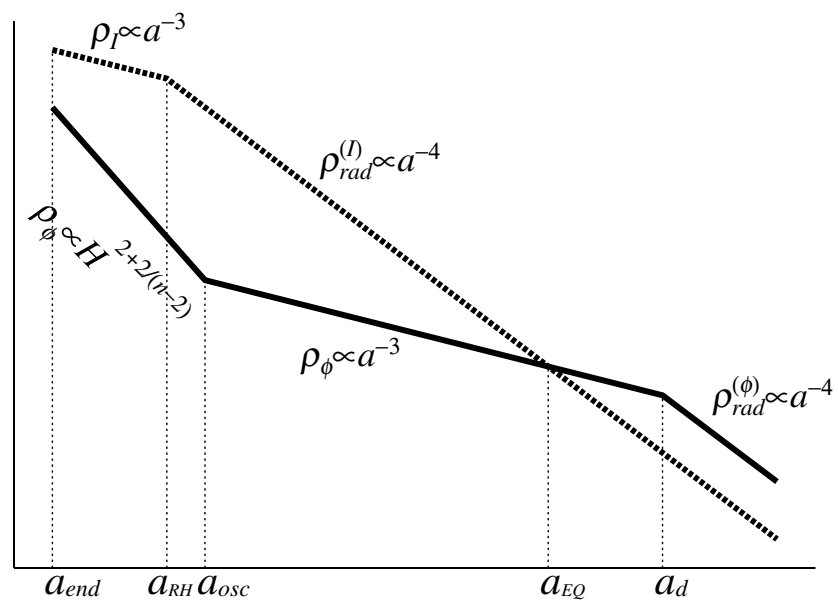

FIG. 1. Evolution of the energy densities in the observable (solid line) and hidden (dotted line) sectors. The subscripts "end," "RH," "osc," "EQ," and "d" refer to, respectively, the end of inflation, reheating by the inflaton decay creating hidden radiation, the beginning of the flat direction oscillation in $m_{\phi}^{2} \phi^{2}$ potential, the equality of the energy densities of $\phi$-oscillation and hidden radiation, and the decay of the flat direction to produce ordinary radiation. 
universe. After its decay into light particles in the hidden sector, the energy density will decrease as $a(t)^{-4}$.

On the other hand, at first the flat direction is dominated by a nonrenormalizable term, and the field follows the boundary of a slow roll regime, $V_{\phi}^{\prime \prime}(\phi) \sim H$, until the supersymmetry breaking mass term begins to dominate the potential when $H \sim m_{\phi} \approx \mathcal{O}(1) \mathrm{TeV}$. The energy density of the inflaton is initially larger than that of the flat direction, as it should be, so the latter never dominates the universe at this stage, since it behaves as $\rho \propto$ $H^{2+2 /(n-2)}$, whereas $\rho \propto H^{2}$ for both the oscillating inflaton and hidden radiation.

From then on, the flat direction field starts oscillating in a quadratic potential with the initial amplitude $\phi_{\text {osc }} \sim\left(m_{\phi} M^{n-3} / \lambda\right)^{1 /(n-2)}$, and the energy density decreases as $a(t)^{-3}$. The field will decay when $H \sim \Gamma_{d}$. For a successful scenario, the flat direction has to dominate the universe at the time of decay (note the difference from the usual curvaton scenarios where the curvaton does not necessarily have to dominate the universe). One can achieve such a situation if the Hubble rate $H_{\mathrm{EQ}}$ at the time when $\rho_{\phi}=\rho_{\text {rad }}^{(I)}$ satisfies $H_{\mathrm{EQ}}>H_{d}$.

In order to evaluate $H_{\mathrm{EQ}}$, let us first assume that the flat direction starts its oscillation after the universe is dominated by hidden radiation $\left(H_{\mathrm{osc}}<H_{\mathrm{RH}}\right)$. This is the case shown in Fig. 1. Imposing the condition $H_{\mathrm{EQ}}>H_{d}$, we get the constraint on $\lambda$ [see Eq. (1)] as

$$
\lambda \lesssim f^{-(n-2) / 2}\left(\frac{m_{\phi}}{M_{\mathrm{P}}}\right)\left(\frac{M}{M_{\mathrm{P}}}\right)^{n-3},
$$

where we take the decay rate of the flat direction to be $\Gamma_{d} \sim f^{2} m_{\phi} / 8 \pi$ [see Eq. (2)].

In the opposite case, where the flat direction oscillations begin while the inflaton oscillations still dominate the universe, i.e., $H_{\mathrm{osc}}>H_{\mathrm{RH}}$, the energy density of the flat direction will evolve as $\rho_{\phi} \propto H^{2}$, while $\rho_{\phi} \propto H^{3 / 2}$ in the hidden radiation-dominated universe. Imposing both $H_{\mathrm{EQ}}>H_{d}$ and $H_{\mathrm{osc}}>H_{\mathrm{RH}}$, we obtain exactly the same constraint as given in Eq. (8).

In addition, we must demand that (a) the amplitude of the flat direction is smaller than the cutoff scale, $\phi_{\text {sr }}<M$, (b) the scale of the inflation is smaller than the Cosmic Background Explorer satellite constraint, $\xi<$ $3 \times 10^{-2} \epsilon^{1 / 4} M_{\mathrm{P}}$, and (c) the energy density of the flat direction is smaller than that of the inflaton during inflation, $V(\phi) / V_{I}<1$ at $\phi=\phi_{\text {sr }}$. These conditions from Eqs. (5)-(7) can be expressed as constraints on $\lambda$, which, respectively, read as

$$
\text { (a) } \lambda \gtrsim P_{\zeta}^{1 / 2} \text {, }
$$

(b) $\quad \lambda \gtrsim\left(10^{3}\right)^{n-3} P_{\zeta}^{(n-2) / 2} \epsilon^{-(n-3) / 2}\left(\frac{M}{M_{\mathrm{P}}}\right)^{n-3}$,
(c) $\lambda \gtrsim P_{\zeta}^{1 / 2}\left(\frac{M}{M_{\mathrm{P}}}\right)^{n-3}$.

Comparing these with the upper bound estimated above in Eq. (8), we find that there are consistent scenarios only for $n \geq 6$, since $P_{\zeta} \sim 10^{-10}$ ( $n=6$ case is marginally consistent). However, we should also take note of the fact that the extra energy density in the universe at the BBN time should be less than about $10 \%$ of the total density, which comes from the fact that the extra neutrino species (or more precisely relativistic degrees of freedom) should contribute $\Delta N_{\nu}<1$. If the oscillation of the flat direction dominates the universe long enough, say $H_{\mathrm{EQ}} \gtrsim$ $10 H_{d}$, this condition is easily satisfied. Therefore, we conclude that only $n \geq 7$ flat directions are consistent with our scenario.

Note that the thermal bath in the hidden sector is completely decoupled from the SM sector, and hence its temperature is expected to be much lower than the temperature in the SM sector. The hidden energy density is given by [19]

$$
\rho_{\mathrm{hid}}=\left(\frac{a_{d}}{a_{\mathrm{EQ}}}\right)^{-1} \rho_{\mathrm{obs}} \sim\left[f^{2}\left(\frac{\lambda M_{\mathrm{P}}}{m_{\phi}}\right)^{4 /(n-2)} \beta\right]^{2 / 3} \rho_{\mathrm{obs}},
$$

where $\beta=1$ for $H_{\mathrm{osc}}<H_{\mathrm{RH}}, \beta=m_{\phi} / H_{\mathrm{RH}}$ for $H_{\mathrm{osc}}>$ $H_{\mathrm{RH}}$, and $M=M_{\mathrm{P}}$ is assumed, so that the hidden radiation can constitute typically at most $10 \%$ of the total energy density (if the hidden sector contains massive particles whose energy density will eventually dominate, one should compare the density with the CDM density).

As is well known, most of the MSSM flat directions carry baryon and/or lepton numbers, and act like the Affleck-Dine (AD) field, which creates baryon (lepton) asymmetry by virtue of the helical motion induced by the A term in the potential $[6,15]$. In the present case the energy density of the AD field dominates the universe, and the baryon-to-entropy ratio would in general become of order unity, which is $10^{10}$ times larger than the value predicted by the BBN [20]. One way out of this impassé is to suppress the phase $\theta$ of the flat direction to be $10^{-10}$, since the baryon number is proportional to $\sin (n \theta) \sim n \theta$. However, in that case the amplitude of the isocurvature fluctuation of flat direction, $\delta \theta / \theta \sim H_{\text {inf }} / \phi \theta$, becomes unacceptably large. Therefore, if the flat direction is to act as a curvaton, it cannot possess any baryon (or lepton) number. More precisely, since the flat direction decays well above the electroweak scale, it can carry baryon and lepton numbers, but not $B-L$. Otherwise, there will be a huge baryonic and/or leptonic isocurvature fluctuation. Unfortunately, no such direction with $n \geq 7$ exists in MSSM.

However, there are two directions which do not receive contributions to A terms from the nonrenormalizable superpotential which lifts the flatness. They are the 
directions $n=7 L L d d d$ (lifted by $H_{u} L L L d d d$ ) and $n=$ $9 Q u Q u Q u e$ (lifted by $Q u Q u Q u H_{d} e e$ ). Since they are lifted by superpotentials of the form $\phi^{n-1} \psi / M^{n-3}$, where $\psi$ is the field other than the flat direction, $\langle\psi\rangle=0$ usually leads to a vanishing A term [21]. Thus, these two directions do not produce any baryon and/or lepton number, and are free of disastrous isocurvature fluctuations. They seem to be excellent candidates for all matter and for the adiabatic density perturbations in models where inflation takes place in a hidden sector.

To summarize, we have discussed a simple scenario where the decay [22] of the $n=7$ or 9 MSSM flat direction creates ordinary matter such as quarks, leptons, and the LSP dark matter, simultaneously providing adiabatic density perturbations. Since the reheating temperature for the ordinary visible radiation comes out to be $\sim 10^{5} \mathrm{GeV}$, baryogenesis can take place at the electroweak phase transition. Moreover, the reheat temperature is low enough to avoid any gravitino problem [23] in the visible sector [24].

Although the situation described in this Letter is rather radical in the sense that the inflaton is now in a completely hidden sector, such an assumption could, in fact, be considered very natural on the basis that the inflaton should anyway couple extremely weakly to the SM. Such "hidden inflation" has the virtue that generation of matter and density perturbations can take place in a sector which is testable in future accelerator experiments.

S. K. is grateful to M. Kawasaki for useful discussions. K.E. is supported partly by the Academy of Finland Grant No. 51433, and A. M. acknowledges Cliff Burgess, Zurab Berezhiani, and Andrew Liddle for helpful discussion.

[1] C. B. Netterfield et al., Astrophys. J. 571, 604 (2002).

[2] A. T. Lee et al., Astrophys. J. 561, L1 (2001).

[3] N.W. Halverson et al., Astrophys. J. 568, 38 (2002); C. Pryke et al., Astrophys. J. 568, 46 (2002).

[4] A. R. Liddle and D. H. Lyth, Cosmological Inflation and Large Scale Structure (Cambridge University Press, Cambridge, 2000).

[5] T. Gherghetta, C. Kolda, and S. P. Martin, Nucl. Phys. B468, 37 (1996).

[6] K. Enqvist and A. Mazumdar, hep-ph/0209244.
[7] K. Enqvist and J. McDonald, Phys. Rev. Lett. 83, 2510 (1999).

[8] K. Enqvist and J. McDonald, Phys. Rev. D 62, 043502 (2000).

[9] M. Kawasaki and F. Takahashi, Phys. Lett. B 516, 388 (2001).

[10] D. H. Lyth and D. Wands, Phys. Lett. B 524, 5 (2002).

[11] K. Enqvist and M. S. Sloth, Nucl. Phys. B626, 395 (2002).

[12] T. Moroi and T. Takahashi, Phys. Lett. B 522, 215 (2001); Phys. Rev. D 66, 063501 (2002); N. Bartolo and A. R. Liddle, Phys. Rev. D 65, 121301 (2002); M. S. Sloth, hep$\mathrm{ph} / 0208241$; D. H. Lyth, C. Ungarelli, and D. Wands, Phys. Rev. D 67, 023503 (2003); K. Dimopoulos and D. H. Lyth, hep-ph/0209180; M. Bastero-Gil et al., hep-ph/ 0211011; T. Moroi and H. Murayama, hep-ph/0211019.

[13] P. Binétruy and G. Dvali, Phys. Lett. B 388, 241 (1996).

[14] M. K. Gaillard, H. Murayama, and K. A. Olive, Phys. Lett. B 355, 71 (1995).

[15] M. Dine, L. Randall, and S. Thomas, Nucl. Phys. B458, 291 (1996).

[16] Thermal corrections due to hidden radiation are negligible, since the flat direction couples to it only gravitationally. On the other hand, after the flat direction begins its oscillation, ordinary radiation is gradually produced by the decay of the flat direction, but its energy density is too small to affect the dynamics of the flat direction.

[17] V. A. Rubakov and M. E. Shaposhnikov, Usp. Fiz. Nauk 166, 5 (1996) [Phys. Usp. 39, 461 (1996)].

[18] G. Jungman, M. Kamionkowski, and K. Griest, Phys. Rep. 267, 195 (1996).

[19] K. Enqvist, A. Jokinen, S. Kasuya, and A. Mazumdar (to be published).

[20] K. A. Olive, G. Steigman, and T. P. Walker, Phys. Rep. 333, 389 (2000).

[21] For a nonminimal Kähler potential, it is possible to have some A terms. In this situation, it might be possible to have a right amount of the baryon asymmetry from the flat direction which acts as a curvaton [19].

[22] Depending on the SUSY parameter values, the flat direction could first fragment to form $Q$ balls.

[23] J. Ellis, J. E. Kim, and D.V. Nanopoulos, Phys. Lett. B 145, 181 (1984); A. L. Maroto and A. Mazumdar, Phys. Rev. Lett. 84, 1655 (2000); R. Kallosh et al., Phys. Rev. D 61, 103503 (2000).

[24] Gravitinos could, however, be produced thermally also by the hidden sector radiation. For low enough reheat temperature in the hidden sector, or for large enough entropy production due to the decay of the flat direction in the visible sector, there should be no gravitino problem [19]. 